\title{
Time efficiency of direct anterior hip arthroplasty compared to postero-lateral approach in elderly patients
}

\author{
Paweł Skowronek ${ }^{1}$, Artur Wojciechowski², Krzysztof Wypniewski ${ }^{1}$, Marcin Sibiński ${ }^{3}$, \\ Michał Polguj ${ }^{4}$, Agnieszka Maksymiuk-Kłos ${ }^{5}$, Paweł Piątkiewicz 5,6
}

\author{
${ }^{1}$ Clinic of Orthopaedics and Traumatology WSZZ Kielce, Jan Kochanowski University, \\ Kielce, Poland \\ ${ }^{2}$ St. George's University Hospitals NHS Foundation Trust, Radiology Department, \\ Blackshaw Road Tooting, United Kingdom \\ ${ }^{3}$ Clinic of Orthopaedics and Paediatric Orthopaedics, Medical University of Lodz, Lodz, \\ Poland \\ ${ }^{4}$ Department of Angiology, Medical University of Lodz, Lodz, Poland \\ ${ }^{5}$ Department of Internal Diseases, Diabetology and Endocrinology, Medical University \\ of Warsaw, Warsaw, Poland \\ ${ }^{6}$ Medical Department, Collegium Medicum, Cardinal Stefan Wyszynski University, \\ Warsaw, Poland
}

Submitted: 18 December 2017

Accepted: 6 April 2018

Arch Med Sci 2021; 17 (1): 106-112

DOI: https://doi.org/10.5114/aoms/86185

Copyright $\odot 2020$ Termedia \& Banach

\section{Abstract}

Introduction: Primary arthroplasty of the hip joint is currently one of the most commonly performed procedures in orthopedics. In Poland we are observing significant changes in the age structure. With the prolonged life more and more elderly patients require musculoskeletal surgery to maintain comfortable and painless mobility. Reducing the duration of the procedure reduces the costs of anesthesiology, surgical and instrument teams, as well as the operating room technical team. The aim of the study was to compare the time required to perform hip joint arthroplasty by the direct anterior approach (DAA) with the postero-lateral approach (PLA) in our hospital.

Material and methods: A retrospective analysis of 559 total and bipolar cemented and cementless hip replacement procedures based on two operative approaches - the minimally invasive DAA over the course of 2 years, and the standard PLA over the course of 3 years - was performed.

Results: Statistically significant differences were observed between the approaches used for cementless total arthroplasty with regard to the mean treatment times: $51.9 \mathrm{~min}$ for the 272 DAA cases, and $78.3 \mathrm{~min}$ for the 190 PLA cases $(p<0.0001)$. For the cementless hemi-arthroplasty procedure, the mean treatment times were $46.9 \mathrm{~min}$ in 36 patients for DAA, and $48.2 \mathrm{~min}$ for 61 patients for PLA $(p=0.57)$.

Conclusions: Minimally invasive DAA significantly shortens the time of the procedure in elderly patients compared to PLA. Further study is needed to analyze other aspects of those two approaches.

Key words: elderly patients, total hip replacement, surgery time, direct anterior approach, posterolateral approach.

\section{Introduction}

Primary arthroplasty of the hip joint is currently one of the most commonly performed procedures in orthopedics [1, 2]. Currently, increasing

\author{
Corresponding author: \\ Agnieszka Maksymiuk-Kłos MD \\ Department of \\ Internal Diseases, \\ Diabetology and \\ Endocrinology \\ Medical University of Warsaw \\ 231 Czermniakowska St \\ 00-416 Warsaw, Poland \\ E-mail: \\ agamaksymiuk@gmail.com
}


numbers of patients and surgical procedures are observed. The effectiveness of operating room (OR) use is affected by several key factors, including the number of operations performed each day, the cost of the treatments themselves and the time required for the patient to be anesthetized and the procedure itself. Reducing the duration of the procedure reduces the cost of the anesthesiology, surgical and instrument teams, as well as the OR technical team. It also reduces the risk of infection by reducing the exposure time of the wounds in the operating theater [3-5]. In addition, the efficiency of the OR would be improved by reducing the time needed for patient preparation and the procedure itself, thus allowing more procedures to be performed.

The direct anterior approach (DAA) and the postero-lateral approach (PLA) are the two most common methods of cementless total arthroplasty in everyday orthopedic practice [6, 7]. Medical observations indicate that DAA is more beneficial for patients' pain perception and recovery of postoperative functions. Hospitalization time and dislocation rate are also shorter in DAA than in PLA [6]. In the work of Bergin et al. it was emphasized that DAA had a shorter operation time than PLA [7].

The age structure of the world's population has changed dramatically in recent years. The number of people over 65 years old (elderly age) in the general population is rapidly increasing. It is connected with new challenges not only for the community but also for modern healthcare. This same problem is observed in Poland. At the end of 2014, the Polish population numbered 38.5 million people. Over 8.5 million people were at least 60 years old (22\% of the population). At the beginning of the 1990 s, this percentage was $15 \%$. It is forecast that the number of elderly people in 2035 (in 20 years) in Poland will rise to 11.4 million (32\%) (almost $1 / 3$ of the population) [8]. Elderly people are characterized by multiple diseases. Cardiovascular diseases, cancers, musculoskeletal system disorders, depression and cognitive impairment are more often observed in this age group. Especially musculoskeletal system disorders significantly reduce patients' autonomy and frequently make normal functioning impossible. Therefore, safe and fast surgical procedures are the most desirable.

The aim of the present study is to compare the time required to perform hip joint arthroplasty by DAA with that of PLA in the hospital of the first author.

\section{Material and methods}

A retrospective analysis of 559 consecutive total and bipolar cemented and cementless hip replacement procedures based on two operative approaches - the minimally invasive DAA, over the course of two years, and the standard postero-lateral approach (PLA), over the course of 3 years - was performed. Types of prostheses are given in Table I. All cementless prostheses, press fit panes, and "hammered" mandrels - monoblock (one-piece) - were used with similar characteristics and implantation technique. Prostheses were used without additional stabilization with screws; in the case of the need for additional stabilization with screws, these patients were excluded. The number of each implants used is also comparable and corresponds to the size of the DAA and PLA groups. For creation of the most representative and comparable groups of patients, only patients with hip joint primary arthrosis were selected for the evaluation, while patients with post-traumatic lesions, primary hip procedures, hip or femoral head fractures and post-articulation requiring any bone reconstruction within the hip joint were excluded. It made it possible to eliminate any additional technical activities required for the initial total knee arthroplasty. For this reason, the types of acetabular components used were also standardized, press fit acetabular components were left (hammered), and no screw-type acetabular components were included in the group of patients (screwin). Both analyzed groups of patients were comparable in terms of age: mean 72.0 years (range: $56-83$ ) vs. 74.6 years (range: $59-87$ ), for DAA and PLA respectively $(p=0.3)$. They were also comparable with regard to body mass index (BMI), mean $25.3 \mathrm{~kg} / \mathrm{m}^{2}$ for DAA compared to $27.9 \mathrm{~kg} / \mathrm{m}^{2}$ for PLA $(p=0.36)$, and sex: 215 female and 93 male vs. 186 female and 65 male for DAA and PLA respectively $(p=0.26)$. From both groups of patients those who had additional stabilizing screws during the implantation of the press fit acetabular components were excluded. It allowed us to obtain the most comparable groups possible with the use of uniform implants.

Patients undergoing the procedure had well controlled blood pressure, and properly monitored blood glucose and electrolytes. There were no patients with advanced renal or hepatic impairment. Heart failure (NYHA I-II) was controlled by oral drugs. All information was retrospectively acquired from the operative protocols.

In total, $96 \%$ of the patients were operated on under spinal anesthesia, with the remaining

Table I. Types of implants used for cementless total arthroplasty

\begin{tabular}{|lcc|}
\hline Stem/cup & DAA & PLA \\
\hline Tri-lock/pinnacle & 68 & 59 \\
\hline Fitmore/trilogy & 34 & 22 \\
\hline Corail/pinnacle & 119 & 78 \\
\hline Polarstem/R3 & 32 & 24 \\
\hline Exceed/Taperlock microplasty & 19 & 7 \\
\hline
\end{tabular}


4\% under general anesthesia. General anesthesia was performed in 10 patients with DAA and 12 patients with PLA. The choice of anesthesia was not dependent on the approach used for the procedure, but on the indications of the anesthesiologist. The measurement of the time needed for the preparation of the patient for the procedure, and for the procedure itself to take place, began at the moment that anesthesia was stopped, irrespective of the type of anesthesia type used.

The time needed for preparation was defined as the period from the start of anesthesia (spinal or general) and the confirmation by the anesthetist that the orthopedic preparations could begin, i.e. positioning the patient on the table, to the moment of completing the first incision. All procedures were performed on operating tables supplied by one vendor. The same instrumental teams assisted for all procedures, using standard orthopedic instrumentation belonging to the OR.

During preparation for the procedure using the PLA approach, the patient lay on the side and was stabilized. The operative field, comprising one limb, was sterilized using a sterile hip package, with one bag on the lower leg and a "U"-type cover.

For the DAA procedure, the patient was placed in a relaxed horizontal position with the hip stabilized against a single support fastened to the table. The two limbs were disinfected and covered to allow hyperextension in the hip joints for both limbs during the procedure [9-12]. A JKT package with two factory-prepared rubber holes per limb was used to prepare the cover. The average preparation times were $6.3 \mathrm{~min}$ for the DAA approach, and 12.2 min for the PLA approach.

The duration of the procedure was measured from the moment of incision until the end of skin stitching and the application of the dressing, with this information being recorded in the operating card. Both total and bipolar cementless arthroplasty were included in the evaluation.

The assessed PLA procedures were performed in the period $2010-2012$ by three surgeons who performed a minimum of 80 procedures a year. Each assessed surgeon had been performing HIP replacement surgery for a minimum of 8 years with no experience with the DAA approach. All doctors performed both total and bipolar hip replacement.

The analyzed group was divided into those who underwent either cemented or cementless procedures for either total or hemi-arthroplasty using the anterior approach (DAA and MIS DAA) during the period 2014-2015. These procedures were performed by two surgeons who perform a minimum of 100 arthroplastic procedures per year using DAA, but not PLA. One of the surgeons had 8 years' experience of hip joint replacement us- ing the DAA and Watson-Jones approach, while the other had been performing the procedure for 12 years. In both groups, the operating teams comprised three doctors: an operator and two assistants.

The PLA was performed by the standard approach, with cutting and stitching of the external rotators, fascia, subcutaneous tissue and skin. No automatic retractors were used beyond the skin. After implantation of all parts of the endoprosthesis tissues were closed in layers. Special care was taken to suture the capsule and piriformis muscle to the greater trochanter. The DAA used the intermuscular space between the tensor fascia lata muscle and the sartorius with no iatrogenic cutting of the muscle tissue. During the preparation of the femur, the operating table was hyperextended by $15^{\circ}$ at the level of the hips. The standard positions of the limbs were changed in favor of making a light 20-degree bend in the knee, and adduction and maximum external rotation of the limb to $90^{\circ}$. This method avoids massive joint capsule release and allows the femur to be exposed using two retractors only. The method did not use direct or indirect tension, braces, traction tables or automatic retractors. When the DAA approach was used, stitches were made only on the fascia, subcutaneous tissue and skin. Neither of the approaches used standard fluoroscopy.

Drains were used for all groups of patients. Procedures which used "staplers" for closing the skin were excluded from the analysis. The skin sutures were performed by the operator or the first assistant. No "cocoon"-type compression bandages were used in any group. The treatments used a variety of uncemented implants: "short" Tri-lock bolts, Fitmore, Taperlock microplasty, or standard Corail or Polarstem systems. Only standard instrumentation was used, as supplied by the manufacturers. All the acetabular cups were press-fit. During bipolar arthroplasty, Corail and ECOFIT spindles were used with their respective heads (Tables I and II). Head sizes were selected by "passage" or caliper measurements.

\section{Statistical analysis}

Before making comparisons of means, the normality of the distributions of the measurable traits was examined by the Kolmogorov compliance test. Because the distributions of the characteristics analyzed in the study in each group were markedly normal, Student's t-test for independent samples was used to compare duration of operation and time of preparation between patients operated on from posterior and anterior approaches for all types of hip replacements. For $2 \times 2$ tables, the $\chi^{2}$ test was used. A $p$-value of less than 0.05 was considered statistically significant. 


\section{Results}

In all treatment groups, shorter treatment and preparation times were observed for DAA: the mean preparation time was $6.3 \mathrm{~min}$, with times ranging from three to $10 \mathrm{~min}$. In contrast, the mean preparation time for the PLA was $12.2 \mathrm{~min}$, ranging from 4 to $15 \mathrm{~min}$ (Table III).

Statistically significant differences were observed between the approaches used for cementless total arthroplasty with regard to the mean treatment times: 51.9 min for the 272 DAA cases, and $78.3 \mathrm{~min}$ for the 190 PLA cases $(p<0.0001)$. The mean time for the procedure was 16 min (34\%) shorter when DAA was used than PLA. In this group, the mean time for the preparation and procedure together was 32 min less (36\% of the total time) for DAA than PLA (Table III). For the cementless hemi-arthroscopic procedure, the mean treatment times were 53 min in 36 patients for DAA, and $61 \mathrm{~min}$ for 61 patients for PLA. This dif-
Table II. Types of implants used for hemi-arthroplasty

\begin{tabular}{|lcc|}
\hline Stem & DAA & PLA \\
\hline Corail & 34 & 42 \\
\hline Ecofit & 2 & 19 \\
\hline
\end{tabular}

ference was not statistically significant $(p<0.57)$ (Table IV).

No significant differences were observed in terms of early deep infection rate $(p=0.12)$ or intraoperative femur fracture $(p=0.9)$ between DAA and PLA. None of the fractures required internal fixation. Postoperative dislocations were more common with PLA ( $p=0.015)$, and temporary meralgia paresthetica was only present with DAA $(p=0.0001)$ (Table V). All cases of meralgia and paresthetica resolved within a couple of weeks after total hip arthroplasty.

Table III. Time periods comparison between DAA and PLA in cementless total hip replacement

\begin{tabular}{|lcccc|}
\hline Parameter & Approach & $\begin{array}{c}\text { Number } \\
\text { of procedures }\end{array}$ & $\begin{array}{c}\text { Average time } \\
\text { min (SD) }\end{array}$ & P-value \\
\hline Preparation time & DAA & 272 & $6.3(1.9)$ & \\
\cline { 2 - 5 } & PLA & 190 & $12.2(2.8)$ & $<0.0001$ \\
\hline Surgery time & DAA & 272 & $51.9(13.6)$ & $<0.0001$ \\
\hline Total procedure time & PLA & 190 & $78.3(20)$ & $<0.0001$ \\
\cline { 2 - 5 } & DAA & 272 & $58.2(13.7)$ & $90.6(20.1)$ \\
\hline
\end{tabular}

$D A A$ - direct anterior approach, PLA - postero-lateral approach.

Table IV. Time periods comparison between DAA and PLA in hemi-arthroplasty

\begin{tabular}{|lcccc|}
\hline Parameter & Approach & $\begin{array}{c}\text { Number } \\
\text { of procedures }\end{array}$ & $\begin{array}{c}\text { Average time } \\
\text { min (SD) }\end{array}$ & P-value \\
\hline Preparation time & DAA & 36 & $6.4(1.7)$ & $<0.000001$ \\
\cline { 2 - 5 } & PLA & 61 & $13.3(3.2)$ \\
\hline Surgery time & DAA & 36 & $46.9(14)$ & 0.57 \\
\hline Total procedure time & PLA & 61 & $48.2(13.1)$ & $53.4(14.1)$ \\
\cline { 2 - 5 } & DAA & 36 & $61.8(14.6)$ & 0.006 \\
\hline
\end{tabular}

DAA - direct anterior approach, PLA - postero-lateral approach.

Table V. Early complications of DAA and PLA

\begin{tabular}{|lccc|}
\hline Complication & DAA (total 308 patients) & PLA (total 251 patients) & $P$-value \\
\hline Deep infection within 90 days & $1(\%)$ & $4(\%)$ & 0.12 \\
\hline Intraoperative femur fractures & $4(\%)$ & $3(\%)$ & 0.9 \\
\hline Postoperative dislocations & $2(\%)$ & $9(\%)$ & 0.015 \\
\hline Meralgia paresthetica & $19(\%)$ & $0(\%)$ & 0.0001 \\
\hline
\end{tabular}

$D A A$ - direct anterior approach, PLA - postero-lateral approach. 


\section{Discussion}

The success of hip replacement depends on many factors. In particular, in the elderly population, it is extremely important to reduce the risk of intra- and post-operative complications for any reason and reduce the time of hospitalization. In this group of patients, different factors may have a negative impact on the treatment course. The elderly are patients with numerous chronic diseases that affect the recovery time as well as a longer life prediction. Often, in the population of people over 65 years of age nutritional deficiencies (mainly deficiencies in building proteins, vitamins and micronutrients) are observed, which further prolongs the healing and regeneration of tissues. It has an impact on increasing the risk associated with the operation itself or the period of hospitalization.

Other factors with an impact on hip replacement are associated with the type of implant used and the preparation and care of the patient both before and after surgery. It primarily rests on the experience and skills of the surgical team, as noted by Matt, Taunton and Mason [1, 9]. Two of the factors determined by and dependent on the operating team are the choice of operational access and length of the procedure. These are important factors in the overall success of hip arthroplasty and are associated with tangible perioperative and postoperative benefits, with regard to both the medical and economic costs [9-12].

The $34 \%$ shortening of the duration of the procedure performed by DAA compared to PLA results in greater OR efficiency and allows a greater number of procedures to be performed. In addition, a shorter stay in the OR and shorter exposure of the wound further reduce the risk of infectious complications [3-5, 13]. Stocks and Self report that a reduction in procedure time is also associated with a reduced risk of contamination for the surgical instruments, the OR, the operative field and the surrounding tissue [3-5, 13-15].

Furthermore, the use of a minimally invasive approach reduces intraoperative and postoperative blood loss, as well as patient hypothermia, as noted by Pavone in a comparison of various types of operative procedure [16]. Improving the reproducibility and ergonomics of activities performed during surgery also helps reduce the risk of technical errors during joint arthroplasty by avoiding primary iatrogenic damage of the original soft tissue $[1,9,16-19]$.

In addition, shortening the mean treatment time, in this case by more than $30 \%$, results in significant reductions in the cost of the operating room and its staff, allowing additional surgery to be performed, or for the work of the operating team to be shortened.
Therefore, the presented comparison offers a compelling case for the use of DAA to shorten the operating time and reduce damage to soft tissue during surgery $[1,9,10,16-20]$. Several authors have reported minimal risk of damage to the muscle using DAA, together with faster rehabilitation and shorter hospitalization time [18-20].

This is another factor which can reduce the risk of post-operative infection and reduce the overall costs of hip joint arthroplasty [2-5, 12, 21]. In our study the rate of infection was lower in DAA than PLA (1\% DAA , 4\% PLA), probably because of the shorter time of surgery and smaller surgery exposure area. General rates of infection in publications are between $0.3 \%$ and $2.8 \%$, in different papers. The infection issue is more complicated than just the type of hip approach. In many cases it depends on wound healing, additional diseases (diabetes, Buerger's disease) and postoperative recovery time. The problem should be investigated further in a different study with follow-up more than 90 days and checking more details than just the approach in the control group.

Wenz et al. [16] report a shorter procedure time, reduced blood loss, shorter hospitalization and shorter rehabilitation time after release when using the minimally invasive approach. It is also associated with no greater risk following joint arthroplasty for standard operative procedures. Higgins and Barlow note a lower risk of hip joint prosthesis dislocation and a significantly shorter hospitalization period in patients treated using DAA compared to PLA [22]. Iwaki and Minoda also report a reduced risk of deep venous thrombosis when using DAA and when the patient is operated on in the horizontal position [23]. These advantages, combined with the significantly shorter operating procedure associated with using DAA, support the wider application of this approach in primary hip joint arthroplasty.

Short time of treatment, small wound surface, minimal soft tissue damage and reduced risk of dislocation can reduce (as Weber et al. reported) the risk of sexual dysfunction after total hip arthroplasty [24]. Undoubtedly, it improves the patient's quality of life.

There are also a lot of studies about femur fracture in total hip arthroplasty. Its incidence ranges from $0.1 \%$ to $4 \%$. Most of them occur 7 to 8 years after the primary implant and 3 to 4 years after the revision of endoprosthesis implantation. The main risk factors are loosening of the stem of the endoprosthesis and osteoporosis. It is interesting that age, sex and obesity do not constitute significant risk factors [25].

In our groups we have only proximal, neck fracture - Table II - it is just the proximal part of the femur (rest of neck), without secondary sur- 
gery obligatory. The stems were stable and the fractures were treated with non-operative techniques -2 crutches for a long time -3 months - without weight bearing. But the patients were excluded from the main compared groups DAA and PLA. Compared to general rates of femur fractures in both groups we were close to the lower limit of $1 \%$.

In the end the dislocation rate comparing papers is absolutely the lowest in DAA, $0-1 \%$ - the dislocation rates by approach are $2-4.1 \%$ for the posterior approach, $3.4 \%$ for the modified Hardinge approach - lateral, and $0.64 \%$ for DAA in our group compared to $3.5 \%$ in PLA - our study $[26,27]$.

This study has some limitations. Firstly, this is a retrospective series of patients. Secondly, the procedure was performed by different surgeons; however, in everyday practice, doctors are trained to perform the procedure in one standard way and it was not possible to find a surgeon who would be equally skilled in both approaches. In addition, although the cohort of patients is heterogenous in terms of anesthesia, etiology, BMI and choice of implant, this is a consequence of the choice of retrospective analysis for the study with a consecutive series of patients. Furthermore, the two groups of patients receiving DAA or PLA were operated on in different time periods; however, this fact should not influence the results and objectivity of the study. Finally, although the experience of the surgeon will also influence the duration of the operation, it is worth noting that all surgeons who performed the procedures as part of the study have a great deal of experience in the field of arthroplasty.

In conclusion, minimally invasive DAA significantly shortens the time of the procedure in elderly patients compared to PLA. Further study is needed to better understand other aspects of those two approaches.

\section{Conflict of interest}

The authors declare no conflict of interest.

\section{References}

1. Matta JM, Shahrdar C, Ferguson TA. Single-incision anterior approach for total hip arthroplasty on an orthopaedic table. Clin Orthop Rel Res 2005; 441: 115-24.

2. Kurtz SM, Roder C, Lau E, et al. International survey of primary and revision total hip replacement. Paper \#365. 56th Annual Meeting of the Orthopaedic Research Society. New Orleans, March 6-9, 2010.

3. Gosden PE, MacGowan AP, Bannister GC. Importance of air quality and related factors in the prevention of infection in orthopaedic implant surgery. J Hosp Infect 1998; 39: 173-80

4. Evans RP. Current concepts for clean air and total joint arthroplasty: laminar airflow and ultraviolet radiation: a systematic review. Clin Orthop Relat Res 2011; 469: 945-53.

5. Chow TT, Yang XY. Ventilation performance in the operating theatre against airborne infection: numerical study on an ultra-clean system. J Hosp Infect 2005; 59: 138-47.

6. Higgins BT, Barlow DR, Heagerty NE, Lin TJ. Anterior vs. posterior approach for total hip arthroplasty, a systematic review and meta-analysis. J Arthroplasty 2015; 30: 419-34.

7. Bergin PF, Doppelt JD, Kephart CJ, et al. Comparison of minimally invasive direct anterior versus posterior total hip arthroplasty based on inflammation and muscle damage markers. J Bone Joint Surg Am 2011; 93 : 1392-8.

8. Główny Urząd Statystyczny. "Population at age 60+. Demographic structure and health." Note prepared for the meeting of the Polish Sejm Commission for Senior Policy concerning. "Information of the Minister of Health on the impact of demographic change and aging society for the organization of the health system and the National Health Program “ (19.02.2016).

9. https://stat.gov.pl/.../ludnosc_w_wieku_60._struktura_demograficzna_i_zdrowie.pdf

10. Taunton MJ, Mason JB, Odum SM, Springer BD. Direct anterior total hip arthroplasty yields more rapid voluntary cessation of all walking aids: a prospective, randomized clinical trial. J Arthroplasty 2014; 29 (9 suppl): 169-72.

11. Berend KR, Lombardi, AV, Seng B. Enhanced early outcomes with the anterior supine intermuscular approach in primary total hip arthroplasty. J Bone Joint Surg Am 2009; 91 Suppl 6: 107-20.

12. Matta JM, Shahrdar C, Ferguson T. Single-incision anterior approach for total hip arthroplasty on an orthopaedic table. Clin Orthop Rel Res 2005; 441: 115-24.

13. Christensen CP, Karthikeyan T, Jacobs CA. Greater prevalence of wound complications requiring reoperation with direct anterior approach total hip arthroplasty. J Arthroplasty 2014; 29: 1839-41.

14. Noble WC. Dispersal of bacteria from human skin. The International Symposium on Contamination Control. Proceedings of the International Symposium on Contamination Control, Copenhagen, Denmark 1976; 16-24.

15. Stocks GW, Self SD, Thompson B, et al. Predicting bacterial populations based on airborne particulates: a study performed in nonlaminar flow operating rooms during joint arthroplasty surgery. Am J Infect Control 2010; 38: 199-204.

16. Dancer SJ, Stewart M, Coulombe C, et al. Surgical site infections linked to contaminated surgical instruments. J Hosp Infect 2012; 81: 231-8.

17. Wenz J, Gurkan I, Jibodh S. Mini-incision total hip arthroplasty: a comparative assessment of perioperative outcomes. Orthop Magazine 2002; 25: 1031-43.

18. Sariali E, Leonard P, Mamoudy P. Dislocation after total hip arthroplasty using Heuter anterior approach. J Arthroplsty 2008; 23: 266-72.

19. Kennon RE, Keggi JM, Wetmore RS, et al. Total hip arthroplasty through a minimally invasive anterior surgical approach. J Bone Joint Surg Am 2003; 85-A Suppl 4: 39-48.

20. Post ZD, Orozco F, Diaz-Ledezma C, Hozack WJ, Ong A. Direct anterior approach for total hip arthroplasty: indications, technique, and results. J Am Acad Orthop Surg 2014; 22: 595-603.

21. Baerga-Varela L, Malanga GA. Rehabilitation after minimally invasive surgery. Minimally Invasive Total Joint Arthroplasty. Springer 2004; 2-5. 
22. Dorr L. Mini-incision for total hip arthroplasty. Pros, cons and experience to date. Proceedings of the 9th Combined Open Meeting Hip Society and AAHKS; 2003 Feb 8; New Orleans, Louisiana; 2003. J Orthop Surg 2004; 12: $19-24$.

23. Chung WK, Liu D, Foo LSS. Mini-incision total hip replacement - surgical technique and early results. J Orthop Surg 2004; 12: 19-24.

24. Iwaki $\mathrm{H}$, Minoda $\mathrm{Y}$, Ikebuchi $M$. Which muscule sparing approach is better, DAA or Antero-lateral in THA, AAOS 2013.

25. Weber P, Schmidutz F, Ficklscherer A, Steinbruck A, Volkmar J, Roland Durr H. Does total joint arthroplasty impair erectile function? Arch Med Sci 2018; 14: 1087-92.

26. Pavelka T, Salášek $M$, Weisová D. Periprosthetic femoral fractures after total hip replacement: our results and treatment complications. Acta Chir Orthop Traumato Cech 2017; 84: 52-8.

27. Blom AW, Rogers M, Taylor AH, Pattison G, Whitehouse S, Bannister GC. Dislocation following total hip replacement. Ann R Coll Surg Engl 2008; 90: 658-62. 